\title{
Combinatorial Logarithm and Point-Determining Cographs
}

\author{
Ji Li* \\ Department of Mathematics \\ Brandeis University \\ Massachusettes, U.S.A. \\ vieplivee@gmail.com
}

Submitted: May 5, 2012; Accepted: Jul 10, 2012; Published: Jul 19, 2012

Mathematics Subject Classifications: 05A15, 05A19

\begin{abstract}
We obtain a reduced form of the "combinatorial logarithm" $\Omega$ by looking at bijections related to connected point-determining cographs and connected co-pointdetermining graphs.
\end{abstract}

\section{Introduction}

The virtual species $\Omega$, referred to as the "combinatorial logarithm" by Bergeron, Labelle, and Leroux in [12, p. 131], is one that uniquely satisfies the combinatorial equality $1+$ $X=\mathcal{E} \circ \Omega$, where $\mathcal{E}$ is the species of sets. The associated series of $\Omega$ are given on [12, p. 131]. In [7] Labelle gave a formula for computing the molecular expression of $\Omega$. Theoretically [12, Proposition 7, p. 122], every virtual species can be written uniquely as the difference between two "real" species $\Phi=\Phi^{+}-\Phi^{-}$, called the reduced form of $\Phi$, where the molecular decompositions of $\Phi^{+}$and $\Phi^{-}$have disjoint terms. To find such two species is generally hard.

In our previous paper $[8,(2.8)]$, we expressed $\Omega$ as the difference between the species $\mathcal{Q}^{c}$ of connected co-point-determining graphs and the species $\mathcal{P}_{\geqslant 2}^{c}$ of connected pointdetermining graphs with more than one vertex. Unfortunately, this is not a reduced form for the obvious reason that the non-zero species $\mathcal{B}^{c}$ of connected bi-point-determining graphs is a subspecies of both $\mathcal{Q}^{c}$ and $\mathcal{P}^{c}$.

The aim of this paper is to further reduce formula $[8,(2.8)]$ by studying the species of point-determining cographs and the connected ones. In Section 2 we review some terminologies in the combinatorial theory of species. Section 3 lists the main results

${ }^{*}$ This work is supported in part by the National Science Foundation Grant Number DUE-0634532. 
of [8] and illustrates the key methods used in [8]. Section 4 discusses point-determining cographs and a further reduced formula (Corollary 10) for expressing the virtual species $\Omega$ as the difference between two subspecies of cographs.

In the end, a list of notations for species mentioned in this paper is given in Section 5.

\section{Combinatorial Theory of Species}

The combinatorial theory of species was initiated by Joyal in [4]. A species is a functor from the category of finite sets with bijections to itself. A species $F$ generates for each finite set $U$ the set $F[U]$ of $F$-structures on $U$, and for each bijection $\sigma: U \rightarrow V$ a bijection $F[\sigma]: F[U] \rightarrow F[V]$, called the transport of $F$-structures along $\sigma$. The symmetric group $\mathfrak{S}_{n}$ acts on the set $F[n]=F[\{1,2, \ldots, n\}]$ by transport of structures. The $\mathfrak{S}_{n}$-orbits under this action are called unlabeled $F$-structures of order $n$. Each species $F$ is associated with three generating series, the exponential generating series $F(x)=\sum_{n \geqslant 0}|F[n]| x^{n} / n !$, the type generating series $\widetilde{F}(x)=\sum_{n \geqslant 0} f_{n} x^{n}$, where $f_{n}$ is the number of unlabeled $F$ structures of order $n$, and the cycle index

$$
Z_{F}=Z_{F}\left(p_{1}, p_{2}, \ldots\right)=\sum_{n \geqslant 0}\left(\sum_{\lambda \vdash n} \operatorname{fix} F[\lambda] \frac{p_{\lambda}}{z_{\lambda}}\right),
$$

where fix $F[\lambda]$ denotes the number of $F$-structures on $[n]=\{1,2, \ldots, n\}$ fixed by $F[\sigma]$ for some $\sigma$ that is a permutation of $[n]$ with cycle type $\lambda, z_{\lambda}$ is the number of permutations in $\mathfrak{S}_{n}$ that commute with a permutation of cycle type $\lambda$, and $p_{\lambda}$ is the power sum symmetric function (see Stanley [3, p. 297]) indexed by the partition $\lambda$ of $n$. The following identities $[12$, p. 18] illustrate the importance of the cycle index in the theory of species.

$$
\begin{aligned}
& F(x)=Z_{F}(x, 0,0, \ldots), \\
& \widetilde{F}(x)=Z_{F}\left(x, x^{2}, x^{3}, \ldots\right) .
\end{aligned}
$$

We apply operations on species [12] to generate new species, and the operations of species translate into operations of the generating series of species systematically. The species operations that are frequently used in this paper are the sum $\Phi+\Psi$, the product $\Phi \Psi$ or $\Phi \cdot \Psi$, and the composition $\Phi(\Psi)$ or $\Phi \circ \Psi$ of species $\Phi$ and $\Psi$.

If $F$ is a species of structures, we denote by $F_{n}$, for nonnegative integers $n$, the species of $F$-structures concentrated on the cardinality $n$ (see [12, p. 30]), and by $F_{\geqslant n}$ the $F$ structures of cardinality at least $n$. Hence $F_{\geqslant n}=F_{n}+F_{n+1}+\cdots$. We usually write $F_{\geqslant 1}$ as $F_{+}$.

A virtual species is a formal difference of species (see [12, p. 121]). Proposition 18 of $[12$, p. 129] asserts that there exists a unique virtual species which we denote by $\Omega$, the virtual species of "connected $(1+X)$-structures" with $1+X=\mathcal{E} \circ \Omega$, or equivalently, $X=\mathcal{E}_{+} \circ \Omega$. Thus $\Omega$ is referred to as the "combinatorial logarithm of the species $1+X$ " (see $\left[12\right.$, p. 131]), or the compositional inverse of $\mathcal{E}_{+}$, also written $\mathcal{E}_{+}^{\langle-1\rangle}$. The associated 
series of $\Omega$ are given on [12, p. 131]. Every virtual species $\Phi$ can be written uniquely in its reduced form

$$
\Phi=\Phi^{+}-\Phi^{-}
$$

where $\Phi^{+}$and $\Phi^{-}$are species with no molecular components in common [12, Proposition 7, p. 122].

A species $M$ is called a molecular species by Yeh [14] if there is only one isomorphism class of $M$-structures. Thus a molecular species is one that is indecomposable under addition. Every species can be expressed uniquely as the sum of molecular species, and this expression is called its molecular decomposition (see [12, p. 141]). For example, the molecular decomposition of the virtual species $\Omega$ starts with

$$
\Omega=X-\mathcal{E}_{2}+\left(X \mathcal{E}_{2}-\mathcal{E}_{3}\right)+\left(X \mathcal{E}_{3}+\mathcal{E}_{2} \circ \mathcal{E}_{2}-X^{2} \mathcal{E}_{2}-\mathcal{E}_{4}\right)+\cdots
$$

We consider in this paper only simple graphs (without loops or multiple edges). A graph $G$ is thought of as an ordered pair $(V, E)$, where $V=V(G)$ is the vertex set of $G$, and $E=E(G)$ is the edge set of $G$, a set of 2-subsets of $V$. Two graphs are called disjoint if they have no common vertices. An unlabeled graph is formally defined as an isomorphism class of graphs, though we think of an unlabeled graph as simply a graph without vertex labels. A graph with no vertices is called empty. The empty graph is not considered as connected. The empty species, denoted by 0 , is defined by $0[U]=\emptyset$ for all $U$. The species of the empty graph is denoted by 1 . The species of the singleton graph is denoted by $X$.

We denote by $\mathcal{K}$ the species of complete graphs, which are graphs in which each pair of vertices are adjacent. The complement of a complete graph is called an edgeless graph. The species of edgeless graphs, which are graphs with isolated vertices, is the same as the species $\mathcal{E}$ of sets. There is a natural transformation $\alpha$ that produces for every finite set $U$ a bijection between $\mathcal{E}[U]$ and $\mathcal{K}[U]$, namely, sending the edgeless graph on $U$ to the complete graph with vertex set $U$. This bijection is carried through the complementation of graphs. The following diagram commutes for any finite sets $U, V$ and any bijection $\sigma: U \rightarrow V:$

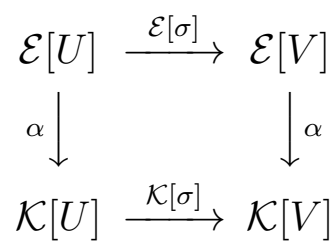

In this case we call these two species isomorphic to each other, denoted $\mathcal{E} \simeq \mathcal{K}$. The general definition of two species being isomorphic to each other is similar. The concept of isomorphism is compatible with the transition to counting series of species (see [12, pp. 1220]). Two isomorphic species essentially possess the "same" combinatorial properties. Henceforth they will be considered as equal in the combinatorial algebra. Thus we write $\Phi=\Psi$ in place of $\Phi \simeq \Psi$, and say there is a combinatorial equality (see [12, p. 21]) between the species $\Phi$ and $\Psi$. 


\section{Enumeration of Bi-Point-Determining Graphs}

The neighborhood of a vertex $v$ is the set of all vertices adjacent to $v$. A point-determining graph (Sumner [13], Bull and Pease [1], also called a mating-type graph, mating graph, or $M$-graph) is a graph in which no two vertices have the same neighborhood. Pointdetermining graphs (both labeled and unlabeled) were counted by Read [9]. Complements of point-determining graphs, which we call co-point-determining graphs (they have also been called "point-distinguishing"), are graphs in which no two vertices have the same closed neighborhood. (The closed neighborhood of a vertex is the vertex together with its neighborhood.) Bi-point-determining graphs (also called "totally point-determining" or "totally supercompact") are both point-determining and co-point-determining.

The notion of superimposition as introduced in [8, Definition 1.2] serves as a bridge bringing together the composition of species and the decomposition of graphs. Let $H_{1}, \ldots, H_{m}$ be graphs with disjoint vertex sets, and let $G$ be a graph with vertex set $\left\{V\left(H_{1}\right), \ldots, V\left(H_{m}\right)\right\}$. We define the superimposition $\left.G\right|_{H_{1}, \ldots, H_{m}}$ of $G$ on $\left\{H_{1}, \ldots, H_{m}\right\}$ to be the graph with vertex set $\bigcup_{i=1}^{m} V\left(H_{i}\right)$ in which $\{u, v\}$ is an edge if it is an edge of some $H_{i}$ or if $u \in V\left(H_{i}\right)$ and $v \in V\left(H_{j}\right)$ for some $i \neq j$, and $\left\{V\left(H_{i}\right), V\left(H_{j}\right)\right\} \in E(G)$.

Figure 1 illustrates the superimposition of a graph $G$ on a set of graphs $\left\{H_{1}, H_{2}, H_{3}\right\}$.

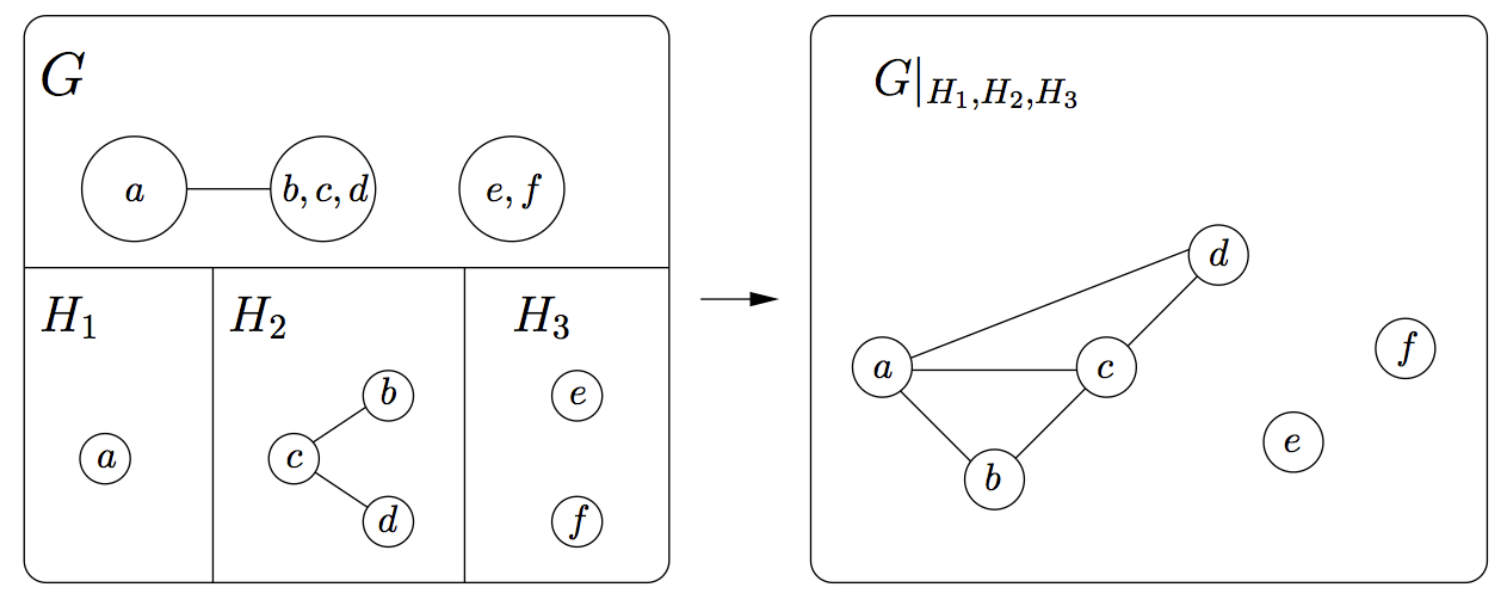

Figure 1: The superimposition $\left.G\right|_{H_{1}, H_{2}, H_{3}}$.

Let $n$ be any positive integer. The edgeless graph of order $n$ is a graph with $n$ isolated vertices, denoted $E_{n}$. The complete graph of order $n$ is a graph with $n$ vertices each pair of which is adjacent to each other, denoted $K_{n}$. Let $\left\{G_{1}, \ldots, G_{n}\right\}$ be a set of nonempty pairwise disjoint graphs. The union of $\left\{G_{1}, \ldots, G_{n}\right\}$ is set to be the superimposition $\left.E_{n}\right|_{G_{1}, \ldots, G_{n}}$, and the join of $\left\{G_{1}, \ldots, G_{n}\right\}$ is set to be the superimposition $\left.K_{n}\right|_{G_{1}, \ldots, G_{n}}$, where the vertex set of $E_{n}$ and $K_{n}$ is $\left\{V\left(G_{1}\right), \ldots, V\left(G_{n}\right)\right\}$.

The operation of superimposition of species of graphs is closely related to composition 
of species. Let $\Phi$ and $\Psi$ be two species of graphs; i.e., for every finite set $U, \Phi[U]$ and $\Psi[U]$ are sets of graphs with vertex set $U$. The species of superimposition $\Phi \diamond \Psi$ is such that $(\Phi \diamond \Psi)[U]$ is the set of all superimpositions $\left.G\right|_{H_{1}, \ldots, H_{m}}$ in which $H_{1}, \ldots, H_{m}$ are $\Psi$-graphs with $\bigcup_{i=1}^{m} V\left(H_{i}\right)=U$ and $G$ is a $\Phi$-graph with vertex set $\left\{V\left(H_{1}\right), \ldots, V\left(H_{n}\right)\right\}$.

Lemma 1. ([8, Lemma 1.4]) Let $\Phi$ and $\Psi$ be species of graphs such that every $\Phi \diamond \Psi$-graph can be expressed uniquely as a superimposition of a $\Phi$-graph on a set of $\Psi$-graphs. Then $\Phi \circ \Psi$ is isomorphic to $\Phi \diamond \Psi$.

We use the combinatorial theory of species [4, 5, 12] as our framework to enumerate point-determining graphs, connected point-determining graphs, and bi-point-determining graphs [8]. The generating series of the species $\mathcal{G}$ of graphs is known (see [12, p. 79] and Robinson [10, p. 334, Theorem 2]). The following theorem gives a way to enumerate point-determining graphs.

Theorem 2. ([8, Theorem 2.2]) For the species $\mathcal{G}$ of graphs, the species $\mathcal{P}$ of pointdetermining graphs, the species $\mathcal{E}_{+}$of nonempty edgeless graphs, the species $\mathcal{Q}$ of copoint-determining graphs, and the species $\mathcal{K}_{+}$of nonempty complete graphs, we have

$$
\mathcal{G}=\mathcal{P} \circ \mathcal{E}_{+}=\mathcal{Q} \circ \mathcal{K}_{+}
$$

The connected point-determining graphs and the connected co-point-determining graphs can be enumerated using the following Theorem.

Theorem 3. ([8, Theorem 2.3]) For the species $\mathcal{P}$ of point-determining graphs, $\mathcal{Q}$ of copoint-determining graphs, $\mathcal{P}^{c}$ of connected point-determining graphs, and $\mathcal{Q}^{c}$ of connected co-point-determining graphs, we have

$$
\mathcal{P}=\mathcal{Q}=(1+X) \cdot\left(\mathcal{E} \circ \mathcal{P}_{\geqslant 2}^{c}\right)=\mathcal{E} \circ \mathcal{Q}^{c} .
$$

A consequence of Theorem 3 is

$$
(1+X) \cdot\left(\mathcal{E} \circ \mathcal{P}_{\geqslant 2}^{c}\right)=\mathcal{E} \circ\left(\Omega+\mathcal{P}_{\geqslant 2}^{c}\right)=\mathcal{E} \circ \mathcal{Q}^{c} .
$$

So [8, Lemma 2.4] gives the following.

Theorem 4. ([8, Equation (2.8)]) We express the virtual species $\Omega$ as the difference between the species $\mathcal{Q}^{c}$ of connected co-point-determining graphs and the species $\mathcal{P}_{\geqslant 2}^{c}$ of connected point-determining graphs with at least two vertices.

$$
\Omega=\mathcal{Q}^{c}-\mathcal{P}_{\geqslant 2}^{c} .
$$

Note that $\mathcal{Q}^{c}-\mathcal{P}_{\geqslant 2}^{c}$ is not the reduced form of $\Omega$, since $\mathcal{Q}^{c}$ share the same molecular components as $\mathcal{P}_{\geqslant 2}^{c}$. Now for any finite set $U$, the intersection $\mathcal{P}^{c}[U] \cap \mathcal{Q}^{c}[U]$ is the set of connected bi-point-determining graphs on $U$, denoted $\mathcal{B}^{c}[U]$. In other words, the species $\mathcal{B}_{\geqslant 2}^{c}$ is a subspecies (see $\left[12\right.$, p. 120]) of both $\mathcal{P}_{\geqslant 2}^{c}$ and $\mathcal{Q}^{c}$. So (3) can be refined into

$$
\Omega=\left(\mathcal{Q}^{c}-\mathcal{B}_{\geqslant 2}^{c}\right)-\left(\mathcal{P}_{\geqslant 2}^{c}-\mathcal{B}_{\geqslant 2}^{c}\right) \text {. }
$$

However, further examination shows that (4) is still not the reduced form of $\Omega$.

A cograph, also called a complement-reducible graph is defined recursively as follows [2]: 
1. A graph on a single vertex is a cograph.

2. For a set of cographs $\left\{G_{1}, \ldots, G_{n}\right\}$, their union $\left.E_{n}\right|_{G_{1}, \ldots, G_{n}}$ is also a cograph.

3. If $G$ is a cograph, then so is its complement.

The species $\mathcal{C}$ of cograghs was enumerated by [8, Lemma 4.2]. The following are unlabeled cographs with no more than four vertices.

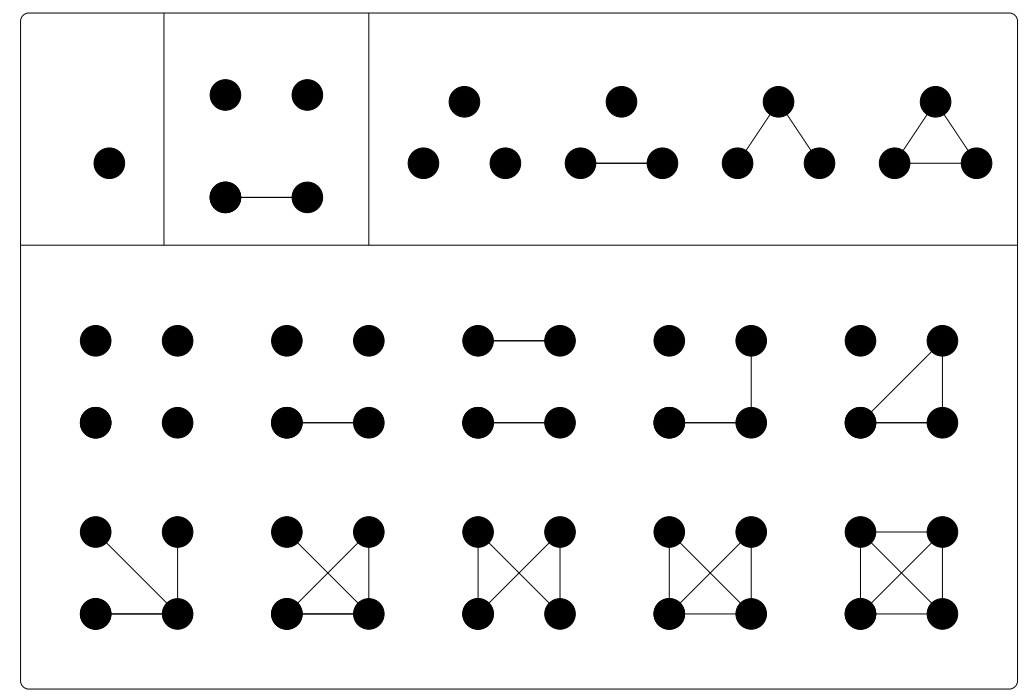

Figure 2: Unlabeled Cographs

The following theorem is the main result of [8].

Theorem 5. ([8, Theorem 4.4]) The species $\mathcal{G}$ of graphs is the composition of the species $\mathcal{B}$ of bi-point-determining graphs and $\mathcal{C}$ of cographs. That is,

$$
\mathcal{G}=\mathcal{B} \circ \mathcal{C} .
$$

Theorem 5 states that every graph can be expressed uniquely as a superimposition of a bi-point-determining graph on a set of cographs. More explicitly, the proof of Theorem 5 gives, for any finite set $U$, a bijection

$$
\alpha_{U}: \mathcal{G}[U] \longrightarrow(\mathcal{B} \diamond \mathcal{C})[U],
$$

between the set $\mathcal{G}[U]$ of all graphs on $U$ and the set of all superimpositions $\left.G\right|_{H_{1}, \ldots, H_{m}}$ such that

1. Each $H_{i}$ is a cograph.

2. The graph $G$ is bi-point-determining.

3. The union of the vertex sets $V\left(H_{i}\right)$ for $i=1,2, \ldots, m$ is equal to the set $U$. 


\section{Connected Point-Determining Cographs}

Now we consider point-determining cographs and co-point-determining cographs. In the literature, the connected point-determining cographs have been enumerated by Moon in $[6]$, where he called them " $\sigma$-networks" or "series-networks", which are series-parallel networks with one or more cut-nodes [11, A058385].

Lemma 6. The species $\mathcal{S}$ of point-determining cographs, $\mathcal{S}^{c}$ of connected pointdetermining cographs, $\mathcal{T}$ of co-point-determining cographs, and $\mathcal{T}^{c}$ of connected co-pointdetermining cographs are related by

$$
\begin{aligned}
\mathcal{S} & =\mathcal{T}, \\
\mathcal{S} & =\mathcal{S}^{c}+\mathcal{T}^{c}-X, \\
\mathcal{S} & =\mathcal{E}_{+} \circ\left(\mathcal{S}^{c}-X\right)+X, \\
\mathcal{T} & =\mathcal{E}_{+} \circ \mathcal{T}^{c},
\end{aligned}
$$

Proof. Since a graph is a cograph if and only if its complement is a cograph, the complement of a point-determining cograph is a co-point-determining cograph, and vice versa. The combinatorial equality $\mathcal{S}=\mathcal{T}$ follows straightforwardly.

On the other hand, $\mathcal{S}-\left(\mathcal{S}^{c}-X\right)$ is the species of the point-determining graphs that are disconnected, whose complements are connected co-point-determining graphs. Therefore, $\mathcal{S}=\mathcal{S}^{c}-X+\mathcal{T}^{c}$.

The proofs of (7) and (8) are similar to that of Theorem 3 in [8].

Lemma 7. The species $\mathcal{S}^{c}$ of connected point-determining cographs and $\mathcal{T}^{c}$ of connected co-point-determining cographs are related by

$$
\begin{aligned}
& \mathcal{S}^{c}=X+\mathcal{E}_{\geqslant 2} \circ \mathcal{T}^{c}, \\
& \mathcal{T}^{c}=X \cdot\left(\mathcal{E} \circ\left(\mathcal{S}^{c}-X\right)\right)+\mathcal{E}_{\geqslant 2}\left(\mathcal{S}^{c}-X\right) .
\end{aligned}
$$

Proof. Let $G$ be a connected point-determining cograph. Then $G$ is either a graph with one vertex or that the complement of $G$ is the union of a set of at least two connected co-point-determining cographs. Equation (9) follows.

Suppose $H$ is a connected co-point-determining cograph. Then the connected components of the complement of $H$ consists of at most one connected component with one vertex, and the rest have to be connected point-determining graphs with more than one vertex. Equation (10) follows.

It follows from (9) and (10) that the species $\mathcal{T}^{c}$ satisfies the following species equation

$$
\mathcal{T}^{c}=X \cdot\left[\mathcal{E} \circ\left(\mathcal{E}_{\geqslant 2} \circ \mathcal{T}^{c}\right)\right]+\mathcal{E}_{\geqslant 2}\left(\mathcal{E}_{\geqslant 2} \circ \mathcal{T}^{c}\right),
$$

which allows us to find the generating series of $\mathcal{T}^{c}$ recursively. Figure 3 shows connected co-point-determining graphs with no more than five vertices.

Next, we will see that the uniqueness of expressing any graph as the superimposition of a bi-point-determining graph on a set of cographs provided by Theorem 5 in [8] gives a way 


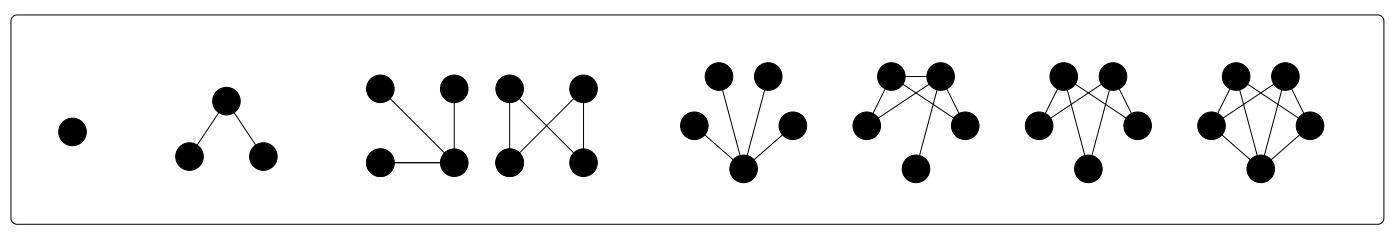

Figure 3: Connected Co-Point-Determining Cographs

to finding new functional equations for connected point-determining graphs and connected co-pointdetermining graphs. We begin with the following Lemma that is straightforward to show.

Lemma 8. The superimposition $\left.G\right|_{H_{1}, H_{2}, \ldots, H_{m}}$ is connected if and only if either $G$ is connected with at least two vertices or $G$ is a single vertex and $H$ is connected.

Theorem 9. For the species $\mathcal{P}^{c}-\mathcal{S}^{c}$ of connected point-determining graphs that are not cographs and $\mathcal{Q}^{c}-\mathcal{T}^{c}$ of connected co-point-determining graphs that are not cographs, we have the following combinatorial equality

$$
\mathcal{P}^{c}-\mathcal{S}^{c}=\mathcal{Q}^{c}-\mathcal{T}^{c}
$$

Proof. Let $U$ be any finite nonempty set, and let

$$
\alpha_{U}: \mathcal{G}[U] \longrightarrow \mathcal{B} \diamond \mathcal{C}[U]
$$

be the bijection in (5) that sends each graph $K$ on $U$ uniquely to a superimposition of a bi-point-determining graph $G$ on a set of cographs $H_{1}, \ldots, H_{m}$ with $\cup_{i=1}^{m} V\left(H_{i}\right)=U$.

Note that because $G$ is bi-point-determining, for any pair $i \neq j \in[m]$, and any vertex $v \in H_{i}$ and $w \in H_{j}, v$ and $w$ can neither have the same neighborhood nor have the same closed neighborhood. Therefore, $K$ is point-determining if and only if each of the $H_{i}$ is point-determining, and that $K$ is co-point-determining if and only if each of the $H_{i}$ is co-point-determining.

Obviously, a graph $K$ on $U$ is a cograph if and only if $\alpha_{U}(K)$ is the superimposition of the form $\left.G\right|_{H_{1}}$ where $G$ is the trivial graph and $H_{1}=K$. In other words, $\alpha_{U}(\mathcal{C}[U])$ is isomorphic to $\mathcal{C}[U]$.

Suppose $K$ is a connected point-determining graph on $U$ that is not a cograph. Then Lemma 8 gives that $\alpha_{U}(K)$ is of the form $\left.G\right|_{H_{1}, \ldots, H_{m}}$ where $G$ is a connected bi-pointdetermining graph with at least two vertices and each of $H_{i}, i=1, \ldots, m$, is a pointdetermining cograph. Therefore,

$$
\alpha_{U}\left(\left(\mathcal{P}^{c}-\mathcal{S}^{c}\right)[U]\right)=\mathcal{B}_{\geqslant 2}^{c} \diamond \mathcal{S}[U]
$$

By a similar argument, we have

$$
\alpha_{U}\left(\left(\mathcal{Q}^{c}-\mathcal{T}^{c}\right)[U]\right)=\mathcal{B}_{\geqslant 2}^{c} \diamond \mathcal{T}[U]
$$


On othe other hand, Equation (6) gives that there exists a bijection

$$
\beta_{U}: \mathcal{B}^{c} \diamond \mathcal{S}[U] \longrightarrow \mathcal{B}^{c} \diamond \mathcal{T}[U]
$$

that is sending each superimposition $\left.G\right|_{H_{1}, \ldots, H_{m}}$ in which each $H_{i}, i=1, \ldots, m$, is a pointdetermining cograph to the superimposition $G_{H_{1}^{\prime}, \ldots, H_{m}^{\prime}}$ where each $H_{i}^{\prime}, i=1, \ldots, m$, is the complement of $H_{i}$.

Thus, for each nonempty finite set $U$, we obtain the bijection

$$
\tau_{U}=\alpha_{U}^{-1} \circ \beta_{U} \circ \alpha_{U}: \mathcal{P}^{c}[U]-\mathcal{S}^{c}[U] \longrightarrow \mathcal{Q}^{c}[U]-\mathcal{T}^{c}[U]
$$

that is sending each connected point-determining graph that is not a cograph uniquely to a connected co-point-determining graph that is not a cograph.

As a consequence of Theorem 4, we obtain a "refined" expression for the virtual species $\Omega$ as the difference of two species.

Corollary 10. The virtual species, so called "combinatorial logarithm" $\Omega$ can be expressed as the difference of the species $\mathcal{T}^{c}$ of connected co-point-determining cographs and the species $\mathcal{S}_{\geqslant 2}^{c}$ of connected point-determining cographs with at least two vertices. That is,

$$
\Omega=\mathcal{T}^{c}-\mathcal{S}_{\geqslant 2}^{c}=X+\mathcal{T}^{c}-\mathcal{S}^{c}
$$

The following Proposition shows that for any finite set $U$ with $\# U>1$, the intersection of the sets $\mathcal{T}^{c}[U]$ and $\mathcal{S}^{c}[U]$ is empty. In other words, there exists no bi-point-determining cographs with more than one vertex.

Proposition 11. The species of bi-point-determining cographs is isomorphic to $X$.

Proof. First of all, the trivial graph is a bi-point-determining cograph. In fact, we will show that there is no bi-point-determining graph with more than one vertex.

Suppose the contrary. We pick $G$ be to one such with the least possible number of vertices. We notice that $G$ cannot be a disjoint union of isolated vertices or a complete graph, since such a cograph is not bi-point-determining. Therefore $G$ must be a disjoin union, or the complement of the disjoint union, of bi-point-determining cographs, at least one of which, say, $H$, has at least two vertices. This contradicts the assumption.

There are still common terms in the molecular expressions of $\mathcal{T}^{c}$ and $\mathcal{S}^{c}$, although these common terms are not bi-point-determining cographs. If we could identify all these common terms, a precise molecular expression of $\Omega$ would be obtained after cancellation. For example, in the following molecular decomposition of $\Omega$ recursively obtained from identities (9) and (10), 


\begin{tabular}{|c|c|c|c|}
\hline$n$ & $\mathcal{T}_{n}^{c}$ & $\mathcal{S}_{n}^{c}$ & $\Omega_{n}=X+\mathcal{T}_{n}^{c}-\mathcal{S}^{c}$ \\
\hline 1 & $X$ & $X$ & $X$ \\
\hline 2 & 0 & $\mathcal{E}_{2}$ & $-\mathcal{E}_{2}$ \\
\hline 3 & $X \mathcal{E}_{2}$ & $\mathcal{E}_{3}$ & $X \mathcal{E}_{2}-\mathcal{E}_{3}$ \\
\hline 4 & $X \mathcal{E}_{3}+\mathcal{E}_{2} \circ \mathcal{E}_{2}$ & $X^{2} \mathcal{E}_{2}+\mathcal{E}_{4}$ & $X \mathcal{E}_{3}+\mathcal{E}_{2} \circ \mathcal{E}_{2}-X^{2} \mathcal{E}_{2}-\mathcal{E}_{4}$ \\
\hline 5 & $\begin{array}{r}X \mathcal{E}_{4}+X^{3} \mathcal{E}_{2} \\
+X \mathcal{E}_{2} \circ \mathcal{E}_{2}+\mathcal{E}_{2} \mathcal{E}_{3}\end{array}$ & $\begin{aligned} X^{2} \mathcal{E}_{3} & +X \mathcal{E}_{2} \circ \mathcal{E}_{2} \\
& +X \mathcal{E}_{2}^{2}+\mathcal{E}_{5}\end{aligned}$ & $\begin{array}{l}X \mathcal{E}_{4}+X^{3} \mathcal{E}_{2}+\mathcal{E}_{2} \mathcal{E}_{3} \\
-X^{2} \mathcal{E}_{3}-X \mathcal{E}_{2}^{2}-\mathcal{E}_{5}\end{array}$ \\
\hline 6 & $\begin{array}{l}X^{3} \mathcal{E}_{3}+X^{2} \mathcal{E}_{2} \circ \mathcal{E}_{2} \\
\quad+2 X^{2} \mathcal{E}_{2}^{2}+X \mathcal{E}_{5} \\
\quad+X \mathcal{E}_{2} \mathcal{E}_{3}+\mathcal{E}_{2} \mathcal{E}_{4} \\
+\mathcal{E}_{2} \circ \mathcal{E}_{3}+\mathcal{E}_{3} \circ \mathcal{E}_{2}\end{array}$ & $\begin{array}{r}X^{4} \mathcal{E}_{2}+X^{2} \mathcal{E}_{4} \\
+X^{2} \mathcal{E}_{2} \circ \mathcal{E}_{2}+3 X \mathcal{E}_{2} \mathcal{E}_{3} \\
+\mathcal{E}_{2} \circ\left(X \mathcal{E}_{2}\right) \\
+\mathcal{E}_{2}\left(\mathcal{E}_{2} \circ \mathcal{E}_{2}\right)+\mathcal{E}_{6}\end{array}$ & $\begin{array}{l}X^{3} \mathcal{E}_{3}+2 X^{2} \mathcal{E}_{2}^{2}+X \mathcal{E}_{5}+\mathcal{E}_{2} \mathcal{E}_{4} \\
+\mathcal{E}_{2} \circ \mathcal{E}_{3}+\mathcal{E}_{3} \circ \mathcal{E}_{2} \\
-X^{4} \mathcal{E}_{2}-X^{2} \mathcal{E}_{4}-2 X \mathcal{E}_{2} \mathcal{E}_{3} \\
-\mathcal{E}_{2} \circ\left(X \mathcal{E}_{2}\right)-\mathcal{E}_{2}\left(\mathcal{E}_{2} \circ \mathcal{E}_{2}\right)-\mathcal{E}_{6}\end{array}$ \\
\hline
\end{tabular}

we notice that in the orders from $n=1$ through $n=4$, there is no repeated terms in $\mathcal{T}_{n}^{c}$ and $\mathcal{S}_{n}^{c}$. When $n=5$, there is one, namely, $X \mathcal{E}_{2} \circ \mathcal{E}_{2}$. When $n=6$, there are two repeated terms $X \mathcal{E}_{2} \mathcal{E}_{3}$ and $X^{2} \mathcal{E}_{2} \circ \mathcal{E}_{2}$. When $n=7$, there are 11 repeated terms in $\mathcal{T}_{7}^{c}$ and $\mathcal{S}_{7}^{c}$, and the situation becomes complicated.

It is desirable to obtain a combinatorial interpretation of further reduced form of the combinatorial logarithm $\Omega$.

\section{$5 \quad$ List of Species}

$\mathcal{C}\left(\mathcal{C}^{c}\right) \quad$ species of (connected) cographs.

$\mathcal{B}\left(\mathcal{B}^{c}\right) \quad$ species of (connected) bi-point-determining graphs.

$\mathcal{E} \quad$ species of edgeless graphs.

$\mathcal{G}\left(\mathcal{G}^{c}\right) \quad$ species of (connected) simple graphs.

$\mathcal{H} \quad$ species of phylogenetic trees.

$\mathcal{K} \quad$ species of complete graphs.

$\Omega=(1+X)^{c}$ the combinatorial logarithm.

$\mathcal{P}\left(\mathcal{P}^{c}\right) \quad$ species of (connected) point-determining graphs.

$\mathcal{Q}\left(\mathcal{Q}^{c}\right) \quad$ species of (connected) co-point-determining graphs.

$\mathcal{R} \quad$ species of special pure 2-trees.

$\mathcal{S}\left(\mathcal{S}^{c}\right) \quad$ species of (connected) point-determining cographs.

$\mathcal{T}\left(\mathcal{T}^{c}\right) \quad$ species of (connected) co-point-determining cographs.

\section{Achknowledgements}

Thanks to Professor Ira Gessel for his support. 


\section{References}

[1] J. J. Bull and C. M. Pease. Combinatorics and variety of mating-type systems. Evolution, 43:667-671, 1989.

[2] D. G. Corneil, H. Lerchs, and L. S. Burlingham. Complement reducible graphs. Discrete Appl. Math., 3:163-174, 1981.

[3] R. P. Stanley. Enumerative Combinatorics. Vol. 2. Cambridge University Press, Cambridge, 1999.

[4] A. Joyal. Une théorie combinatoire des séries formelles. Adv. in Math., 42:1-82, 1981.

[5] A. Joyal. Foncteurs analytiques et espèces de structures. In G. Labelle and P. Leroux, editors, Lecture Notes in Mathematics, volume 1234 of Proceedings, Montreal, Québec, Canada, 1985, pages126-159, Berlin, Heidelberg, and New York, 1986. SpringerVerlag.

[6] J. W. Moon. Some enumerative results on series-parallel networks. Ann. Disc. Math., 33:129-226, 1987.

[7] G. Labelle. New combinatorial computational methods arising from pseudosingletons. DMTCS, AJ:247-258, 2008.

[8] I. Gessel and J. Li. Enumeration of point-determining graphs. J. Combin. Theory Ser. A., 118(2):591-612, 2011.

[9] R. C. Read. The enumeration of mating-type graphs. In Research Report CORR, volume 38. Department of Combinatorics and Optimization, University of Waterloo, 1989.

[10] R. W. Robinson. Enumeration of non-separable graphs. J. Combin. Theory, 9:327356, 1970.

[11] N. Sloane. The Online Encyclopedia of Integer Sequences.

[12] F. Bergeron, G. Labelle, and P. Leroux. Combinatorial Species and Tree-Like Structures. volume 67 of Encyclopedia of Mathematics and its Applications. Cambridge University Press, Cambridge, 1998. Translated from the 1994 French original by Margaret Readdy.

[13] D. P. Sumner. Point determination in graphs. Disc. Math., 5:179-187, 1973.

[14] Y. N. Yeh. On the combinatorial species of Joyal. PhD thesis, State University of New York at Baffalo, 1985. 